\title{
Pseudoquiste pancreático en paciente pediátrico, abordaje laparoscópico
}

\author{
Pancreatic pseudocyst in pediatric patient, laparoscopic approach \\ Sandra Gabriela Ayala Hernández, ${ }^{*}$ Guadalupe Kristal Peña Portillo, ${ }^{*}$ Erwin Iván Marín Pardo, ${ }^{*}$ \\ Guadalupe del Carmen Pérez Torres, ${ }^{*}$ Aaron Zaraín Rodríguez* \\ * Cirugía General, Hospital Universitario de Puebla.
}

\section{RESUMEN}

Introducción: El pseudoquiste pancreático es la colección más frecuente del páncreas. En la población pediátrica, la causa más frecuente es secundaria a traumatismo. La indicación de tratamiento depende de los síntomas y tamaño del pseudoquiste. Caso clínico: Paciente femenino de 17 años con antecedente de pancreatitis de origen metabólico que presenta saciedad temprana y dolor en epigastrio. Se realiza tomografía con evidencia de pseudoquiste. Se decide efectuar cistogastroanastomósis laparoscópica. Conclusión: La cistogastroanastomosis laparoscópica confiere mayores ventajas por su menor invasión, en comparación con el abordaje abierto.

Palabras clave: Pseudoquiste pancreático, cistogastroanastomosis, abordaje laparoscópico.

\section{ABSTRACT}

Introduction: Pancreatic pseudocyst is the most frequent pancreatic collection. In pediatric population the most frequent cause is traumatism. The indication for management depends on symptoms and pseudocyst dimensions. Case report: 17 years old female with previous metabolic pancreatitis, with current symptomatology of early satiety and epigastric pain. Computed tomography shows pseudocyst. Laparoscopic cystogastrostomy is performed. Conclusion: The laparoscopic cystgastrostomy confers greater advantages because of it's least invasion compared to open approach.

Keywords: Pancreatic pseudocyst, cystgastrostomy, laparoscopic approach

\section{INTRODUCCIÓN}

De acuerdo con la clasificación de Atlanta, las colecciones pancreáticas o peripancreáticas se pueden catalogar en: Colecciones agudas, pseudoquiste agudo y crónico, y absceso pancreático. ${ }^{1}$ La más frecuente de todas es el pseudoquiste. Los pseudoquistes son colecciones que están delimitadas por tejido fibroso y pueden contener detritus necróticos o sangre. ${ }^{2}$ El desarrollo de pseudoquistes es una complicación de la pancreatitis aguda, la pancreatitis crónica y en traumatismos pancreáticos. ${ }^{3}$ En los pacientes pediátricos, la causa más frecuente de formación de pseudoquiste es secundaria a traumatismo. ${ }^{4}$ La mayoría de los pseudoquistes en pacientes pediátricos se resuelven de manera espontánea sólo con tratamiento conservador. Aquellos casos que persisten con sintomatología (dolor abdominal, saciedad temprana, pérdida de peso o fiebre) por seis semanas o más, aumento de tamaño (mayor de 6 
cm) o presencia de complicaciones (infección, obstrucción gástrica o biliar, trombosis, rotura), por lo general requieren tratamiento intervencionista..$^{5-7}$ Dicho tratamiento incluye el abordaje endoscópico, laparoscópico o abierto. El tratamiento quirúrgico tiene una tasa de recurrencia de $5 \%$, entre los abordajes se encuentran la cistogastroanastomosis, cistoduodenoanastomosis y cistoyeyunoanastomosis, siendo la primera la que se utiliza con más frecuencia, tanto de forma laparoscópica como abierta. El abordaje laparoscópico se asocia a 93\% de éxito en el drenaje del pseudoquiste, con una recurrencia de $2.5 \%$ y menos de $2 \%$ de complicaciones. ${ }^{8,9}$

\section{PRESENTACIÓN DEL CASO}

Mujer de 17 años, acude a la consulta externa de cirugía general por presentar dolor abdominal localizado en epigastrio y mesogastrio de un mes de evolución, intensidad 7/10 en una escala visual análoga (EVA), se exacerba después de la ingesta de alimentos y presenta saciedad temprana, se agrega emesis de contenido gastroalimentario en dos ocasiones. Cuenta con el antecedente de pancreatitis aguda leve de origen metabólico meses previos a su padecimiento actual. A la exploración física: dolor a la palpación profunda en epigastrio, Murphy ausente; resto de la exploración sin alteraciones. Se realizan estudios de laboratorio: Hemoglobina $12.4 \mathrm{~g} / \mathrm{dL}$, hematocrito 38\%, leucocitos $6.20 \times 10^{3} / \mu \mathrm{L}$, plaquetas $259 \times 10^{3} / \mu \mathrm{L}$, glucosa $103 \mathrm{mg} / \mathrm{dL}$, creatinina $0.52 \mathrm{mg} / \mathrm{dL}$, sodio $142 \mathrm{mEq} / \mathrm{L}$, potasio $3.6 \mathrm{mEq} / \mathrm{L}$, cloro $109 \mathrm{mEq} / \mathrm{L}$, bilirrubina total $0.5 \mathrm{mg} /$ $\mathrm{dL}$, fosfatasa alcalina $83 \mathrm{U} / \mathrm{L}$, amilasa $59 \mathrm{U} / \mathrm{L}$, lipasa $33 \mathrm{U} / \mathrm{L}$.

La tomografía de abdomen contrastada muestra páncreas en situación normal, con presencia de lesión quística dependiente de cuerpo y cola, con dimensiones de $105 \times$ $90 \mathrm{~mm}$, de contenido homogéneo, sin captación de medio de contraste, clasificándose como pseudoquiste (Figura 1).

Debido a los antecedentes de la paciente y por los estudios de gabinete, se decide realizar abordaje laparoscópico.
Se colocan cuatro puertos, se hace disección hasta la transcavidad de los epiplones, se identifican pseudoquiste, se drenan $300 \mathrm{~cm}^{3}$ aproximadamente de características turbias; posteriormente, se hace incisión en cara anterior y posterior del estómago, se efectúa cistogastroanastomosis mecánica con engrapadora lineal y la cara anterior del estómago con PDS 2-0 (Figuras 2 y 3). Se realiza endoscopía transoperatoria con prueba neumática y administración de azul de metileno sin evidencia de fuga por la anastomosis. Se coloca drenaje cerrado dirigido hacia curvatura mayor, se retiran trocares bajo visión directa y se da por finalizado el tiempo quirúrgico.

Se deja en ayuno a la paciente durante 24 horas con vigilancia de gastos a través de sonda nasogástrica y drenaje abdominal, sin evidencia de fuga anastomótica, se reinicia la vía oral con adecuada tolerancia, se da de alta tres días después.

\section{DISCUSIÓN}

El pseudoquiste es la complicación más frecuente posterior a un cuadro de pancreatitis aguda sin importar la etiología de la misma. Se resuelve de forma espontánea en la mayoría de los casos; sin embargo, cuando muestra sintomatología como saciedad temprana, dolor abdominal o pérdida de peso, se debe valorar de forma integral al paciente, apoyándonos en estudios de imagen o endoscopía.

A pesar de los avances en el tratamiento de los pseudoquistes pancreáticos, ya sea por abordaje percutáneo guiado por imagen o de forma endoscópica, la mejor opción de tratamiento en pacientes sin recidivas es el abordaje quirúrgico, abierto o laparoscópico, mostrando menor tasa de recurrencia en comparación con otras técnicas.

La cistogastroanastomosis es el tratamiento de elección, ya que tiene bajas tasas de recurrencia, el abordaje laparoscópico comparado con la técnica abierta tiene ventajas en cuanto a menor tiempo de recuperación, menos días
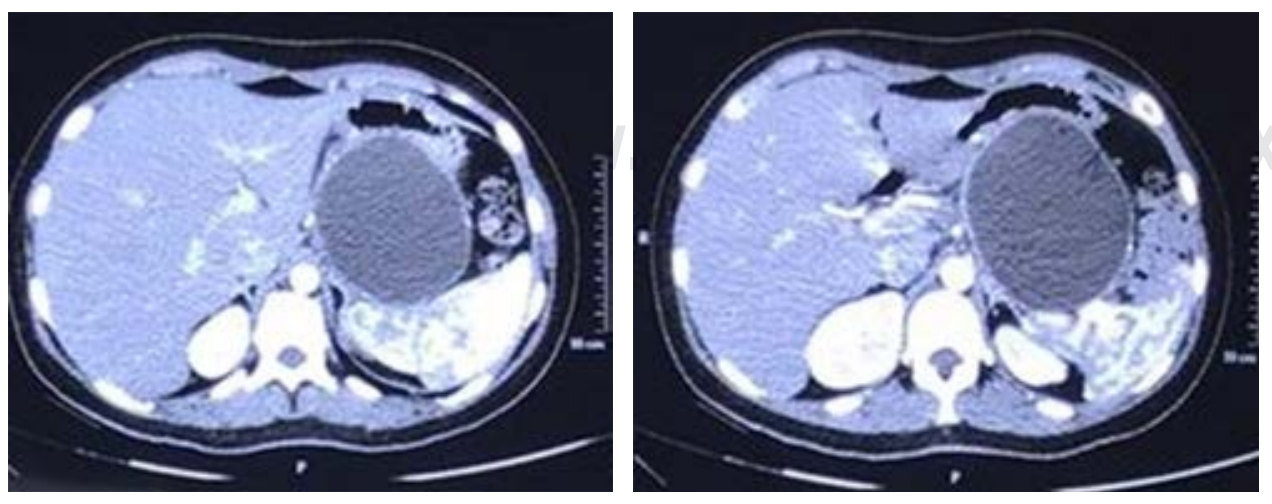

Figura 1:

Tomografía axial computarizada de abdomen contrastada, donde se evidencia pseudoquiste pancreático. 


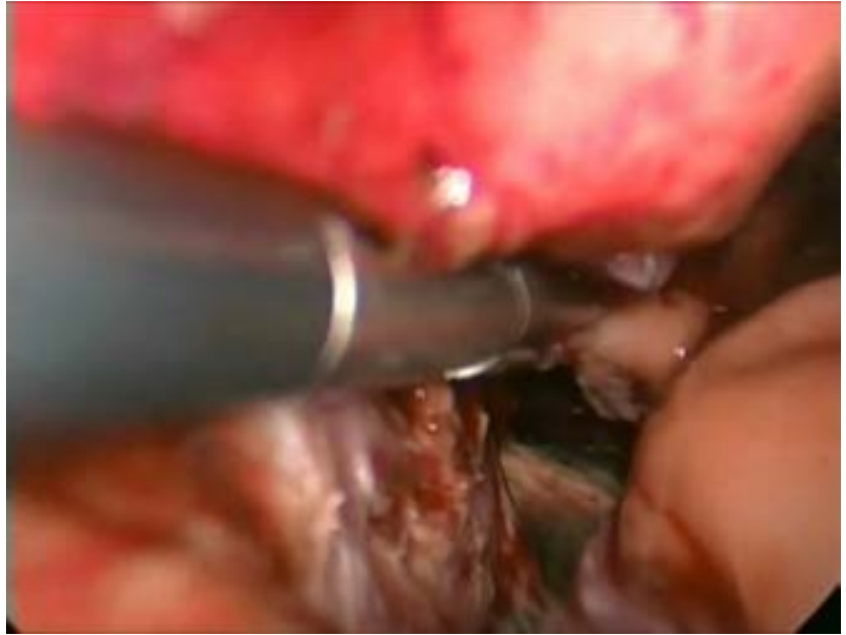

Figura 2: Cavidad del pseudoquiste con presencia de material necrótico en su interior.

de estancia hospitalaria, reincorporación de la vía enteral de forma precoz y menor dolor posoperatorio.

\section{CONCLUSIÓN}

La cistogastroanastomosis laparoscópica como tratamiento definitivo del pseudoquiste pancreático se considera una opción adecuada para el drenaje continuo hacia la cavidad gástrica, ya que presenta una tasa baja de recidiva en comparación con otras técnicas. Al contrastar con el abordaje abierto, se evidencia menor morbimortalidad, mejor aceptación del paciente, e inicio temprano de la vía oral, como se demostró en este caso clínico.

\section{REFERENCIAS}

1. Bradley EL. A clinically based classification system for acute pancreatitis. Summary of the International Symposium on Acute Pancreatitis, Atlanta, Ga, September 11 through 13, 1992. Arch Surg. 1993; 128: 586-590.

2. Patel PA, Gibson C, Minhas KS, Stuart S, De Coppi P, Roebuck DJ. Pancreatic pseudocyst drainage in children by image-guided cystogastrostomy and stent insertion. Pediatr Radiol. 2019; 49: 1816-1822.

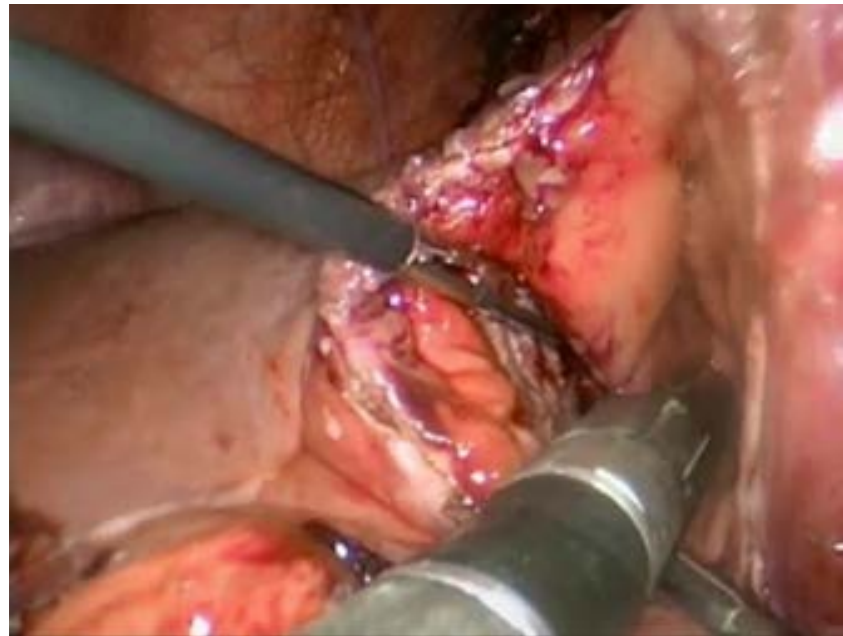

Figura 3: Cistogastroanastomosis mecánica.

3. Al-Shanafey S, Shun A, Williams S. Endoscopic drainage of pancreatic pseudocysts in children. J Pediatr Surg. 2004; 39: 1062-1106.

4. Saad DF, Gow KW, Cabbabe S, Heiss KF, Wulkan ML. Laparoscopic cystogastrostomy for the treatment of pancreatic pseudocysts in children. J Pediatr Surg. 2005; 40: e13-e17.

5. Jazrawi SF, Barth BA, Sreenarasimhaiah J. Efficacy of endoscopic ultrasound-guided drainage of pancreatic pseudocysts in a pediatric population. Dig Dis Sci. 2011; 56: 902-908.

6. Yachha SK, Chetri K, Saraswat VA, Baijal SS, Sikora SS, Lal $\mathrm{R}$ et al. Management of childhood pancreatic disorders: $\mathrm{A}$ multidisciplinary approach. J Pediatr Gastroenterol Nutr. 2003; 36: 206-212.

7. Crisanto-Camposa BA, Arce-Liévano E, Cárdenas-Lailson LE, Romero-Loera LS, Rojano-Rodríguez ME, Gallardo-Ramírez MA. Manejo laparoscópico de los seudoquistespancreáticos: experiencia de un hospital general en la Ciudad de México. Revista de Gastroenterología de México. 2015; 80: 198-204.

8. Agalianos C, Passas I, Sideris I, Davides D, Dervenis C. Review of management options for pancreatic pseudocysts. Transl Gastroenterol Hepatol. 2018; 3: 18.

9. Makris KI, St. Peter SD, Tsao KJ, Ostlie DJ. Laparoscopic intragastric stapled cystgastrostomy of pancreatic pseudocyst in a child. J Laparoendosc Adv Surg Tech A. 2008; 18: 771-773. 